\title{
ON CERTAIN SUBCLASS OF MULTIVALENT ANALYTIC FUNCTIONS ASSOCIATED WITH ERDELYI-KOBER TYPE INTEGRAL OPERATOR
}

\author{
R.M. El-Ashwah, A.Y. Lashin, A.E. El-Shirbiny
}

Abstract. In this paper, we introduce a certain subclasses of multivalent uniformly starlike analytic functions by making use of Erdeyi-Kober type integral operator. Further, we determine coefficient estimates and Holder's inequality results. Also, results for family of class preserving integral operators are obtaind for the class $U S_{p}^{*} T(n, a, c, \mu ; \alpha, \beta)$.

2010 Mathematics Subject Classification: 30C45.

Keywords: $P$-valent analytic functions, Holder's inequality, uniformly starlike, uniformly convex, Erdeyi-Kober type integral operator.

\section{INTRODUCTION}

Let $A(p, n)$ denote the class of functions of the form

$$
f(z)=z^{p}+\sum_{k=n}^{\infty} a_{k+p} z^{k+p} \quad(n, p \in \mathbb{N}=\{1,2, \ldots\}),
$$

which are analytic and p-valent in open unit disc $U=\{z: z \in \mathbb{C},|z|<1\}$. Also, we note that $A(1,1)=A$, that is the class of analytic univalent functions.

A function $f \in A(p, n)$ is said to be in the class $S(p, n, \alpha)$ of p-valent starlike functions of order $\alpha$ if it satisfies the condition

$$
\operatorname{Re}\left(\frac{z f^{\prime}(z)}{f(z)}\right)>\alpha \quad(z \in U ; 0 \leq \alpha<p) .
$$

A function $f \in A(p, n)$ is said to be in the class $K(p, n, \alpha)$ of p-valent convex functions of order $\alpha$ if it satisfies the condition

$$
\operatorname{Re}\left(1+\frac{z f^{\prime \prime}(z)}{f^{\prime}(z)}\right)>\alpha \quad(z \in U ; 0 \leq \alpha<p) .
$$

The classes $S(p, n, \alpha)$ and $K(p, n, \alpha)$ were studied by Owa [18]. The class $S^{*}(p, \alpha)=$ $S(p, 1, \alpha)$ was considered by Patil and Thakare [19]. 
R.M. El-Ashwah, A.Y. Lashin, A.E. El-Shirbiny - On Certain Subclass ...

We denote by $T(p, n)$ the subclass of $A(p, n)$ consisting of functions of the form

$$
f(z)=z^{p}-\sum_{k=n}^{\infty} a_{k+p} z^{k+p} \quad\left(a_{k+p} \geq 0 ; n, p \in \mathbb{N}=\{1,2, \ldots\}\right),
$$

and define two further classes $T^{*}(p, n, \alpha)$ and $C(p, n, \alpha)$ by

$$
T^{*}(p, n, \alpha)=S(p, n, \alpha) \cap T(p, n), \quad C(p, n, \alpha):=K(p, n, \alpha) \cap T(p, n) .
$$

Further, the classes

$$
T^{*}(p, \alpha)=S^{*}(p, \alpha) \cap T(p, n), \quad C(p, \alpha):=K(p, \alpha) \cap T(p, n) .
$$

The function $f(z) \in T(p, n)$ given by (4) is said to be $\beta$-uniformly starlike of order $\alpha(-p \leq \alpha<p)$ and $\beta \geq 0$ denote by $U S_{p}^{*} T(n, \alpha, \beta)$ if and only if

$$
\operatorname{Re}\left(\frac{z f^{\prime}(z)}{f(z)}-\alpha\right)>\beta\left|\frac{z f^{\prime}(z)}{f(z)}-p\right| \quad(z \in U) .
$$

Also, function $f(z)$ is said to be $\beta$-uniformly convex of order $\alpha$ denoted by $U C_{p} V(n, \alpha, \beta)$ [10] if and only if

$$
\operatorname{Re}\left(1+\frac{z f^{\prime \prime}(z)}{f^{\prime}(z)}-\alpha\right)>\beta\left|\frac{z f^{\prime \prime}(z)}{f^{\prime}(z)}-(p-1)\right| \quad(z \in U) .
$$

Note that, the classes $U S_{1}^{*} T(1, \alpha, \beta)=U S^{*} T(\alpha, \beta)$ and $U C_{1} V(1, \alpha, \beta)=U C V(\alpha, \beta)$ are introduced and studied by Bharati et al. [4]. In particular, the classes $U C V(0,1)$ and $U C V(0, \beta)$ were introduced by Goodman [7] and Kanas and Wisniowska [9].

Definition 1. [2] For $f \in A(p, n), p, n \in \mathbb{N}, \mu>0, a, c \in \mathbb{C}, \operatorname{Re}(a) \geq-\mu p$ and $\operatorname{Re}(c-a)>0$, El-Ashwah and Drbuk define the differ-integral operator which called Erdelyi-Kober type integral operator $I_{p, \mu}^{a, c}: A(p, n) \longrightarrow A(p, n)$ as follows

$$
I_{p, \mu}^{a, c} f(z)=z^{p}+\sum_{k=n}^{\infty} \Psi_{p, \mu}^{a, c}(k) a_{k+p} z^{k+p},
$$

where

$$
\Psi_{p, \mu}^{a, c}(k)=\frac{\Gamma(c+\mu p)}{\Gamma(a+\mu p)} \frac{\Gamma(a+\mu(k+p))}{\Gamma(c+\mu(k+p))} .
$$


R.M. El-Ashwah, A.Y. Lashin, A.E. El-Shirbiny - On Certain Subclass ...

If $a=c$, then we have $I_{p, \mu}^{a, a} f(z)=f(z)$. It easily to verify that,

$$
z\left(I_{p, \mu}^{a, c} f(z)\right)^{\prime}=\frac{a+\mu p}{\mu} I_{p, \mu}^{a+1, c} f(z)-\frac{a}{\mu} I_{p, \mu}^{a, c} f(z) .
$$

We also note that the operator $I_{p, \mu}^{a, c} f(z)$ generalizes several previously studied familiar operators and we will mention some of the interesting particular cases as follows:

(1) For $p=1$, we can obtain the operator $I_{\mu}^{a, c} f(z)$ defined in [11, ch.5] (see also [20] and [21, with $m=0])$;

(2) For $a=\beta, c=\beta+1$ and $\mu=1$, we obtain the familiar integral operator $I_{\beta, p} f(z)$ $(\beta>-p)$ which studies by Saitoh et al. [23];

(3) For $a=\beta, c=\alpha+\beta-\gamma+1$ and $\mu=1$, we obtain the operator $R_{\beta, p}^{\alpha, \gamma} f(z)(\gamma$ $>0 ; \alpha \geq \gamma-1 ; \beta>-1$ ) studied by Aouf et al. [1];

(4) For $p=1, a=\beta, c=\alpha+\beta$ and $\mu=1$, we obtain the operator $Q_{\beta}^{\alpha} f(z)$ $(\alpha \geq 0, \beta>-1)$ studied by Jung et al. [8];

(5) For $p=1, a=\alpha-1, c=\beta-1$, and $\mu=1$, we obtain the operator $l(\alpha, \beta) f(z)$ $\left(\alpha, \beta \in \mathbb{C} \backslash \mathbb{Z}_{0}, \mathbb{Z}_{0}=\{0,-1,-2, \ldots .\right.$.$\} studied by Carlson and Shafer [5];$

(6) For $p=1, a=\rho-1, c=\ell$ and $\mu=1$, we obtain the operator $I_{\rho, \ell} f(z)(\rho>0$; $\ell>-1)$ studied by Choi et al. [6];

(7) For $p=1, a=\alpha, c=0$ and $\mu=1$, we obtain the operator $D^{\alpha} f(z)(\alpha>1)$ studied by Ruscheweyh [22];

(8) For $p=1, a=1, c=n$ and $\mu=1$, we obtain the operator $I_{n} f(z)\left(n \in N_{0}\right)$ studied by Noor and Noor [17]; and Noor [16];

(10) For $p=1, a=\beta, c=\beta+1$ and $\mu=1$, we obtain the integral operator $I_{\beta, 1}$ which studied by Bernardi [3];

(11) For $p=1, a=1, c=2$ and $\mu=1$, we obtain the integral operator $I_{1,1}=I$ which studied by Libera [12] and Livingston [14].

Now, we introduced a new subclasses of $p$-valent functions and discussed some interesting geometric properties of this generalized function class. 
R.M. El-Ashwah, A.Y. Lashin, A.E. El-Shirbiny - On Certain Subclass ...

Definition 2. A function $f \in A(p, n)$ is said to be in the class $U S_{p}^{*}(n, a, c ; \mu, \alpha, \beta)$ if it satisfies the inequality

$$
\operatorname{Re}\left(\frac{z\left(I_{p, \mu}^{a, c} f(z)\right)^{\prime}}{I_{p, \mu}^{a, c} f(z)}-\alpha\right)>\beta\left|\frac{z\left(I_{p, \mu}^{a, c} f(z)\right)^{\prime}}{I_{p, \mu}^{a, c} f(z)}-p\right|,(z \in U),
$$

which is equivalent to

$$
\operatorname{Re}\left(\frac{I_{p, \mu}^{a+1, c} f(z)}{I_{p, \mu}^{a, c} f(z)}-\frac{a+\alpha \mu}{a+\mu p}\right)>\beta\left|\frac{I_{p, \mu}^{a+1, c} f(z)}{I_{p, \mu}^{a, c} f(z)}-1\right|, \quad(z \in U)
$$

for some $-p \leq \alpha<p, \beta \geq 0, p, n \in \mathbb{N}, \mu>0, a, c \in \mathbb{C}, \operatorname{Re}(a) \geq-\mu p$ and $\operatorname{Re}(c-a)>0$.

Furthermore, we define the class $U S_{p}^{*} T(n, a, c ; \mu, \alpha, \beta)$ by $U S_{p}^{*}(n, a, c ; \mu, \alpha, \beta) \cap T(p, n)$.

The main object of this work is to determine coefficient estimates for the analytic functions class $U S_{p}^{*} T(n, a, c ; \mu, \alpha, \beta)$. We study some interesting Holder's inequality for the class $U S_{p}^{*} T(n, a, c ; \mu, \alpha, \beta)$. Also, the family of class preserving integral operators for functions $f$ in the class $U S_{p}^{*} T(n, a, c ; \mu, \alpha, \beta)$ are obtained.

\section{Coefficient Inequalities}

Unless otherwise mention, we assume in the reminder of this paper that $\mu>0$, $a, c \in R, a>-\mu p,(a-c)>0,-p \leq \alpha<p, \beta \geq 0, p, n \in \mathbb{N}$. First, we give a coefficients inequality for the class $U S_{p}^{*}(n, a, c ; \mu, \alpha, \beta)$.

Theorem 1. A sufficient condition for a function $f(z)$ of the form (1) to be in $U S_{p}^{*}(n, a, c ; \mu, \alpha, \beta)$ is

$$
\sum_{k=n}^{\infty}[k(1+\beta)+(p-\alpha)] \Psi_{p, \mu}^{a, c}(k)\left|a_{k+p}\right| \leq(p-\alpha)
$$

where

$$
\Psi_{p, \mu}^{a, c}(k)=\frac{\Gamma(c+\mu p)}{\Gamma(a+\mu p)} \frac{\Gamma(a+\mu(k+p))}{\Gamma(c+\mu(k+p))} .
$$

Proof. It is sufficient to show that

$$
\beta\left|\frac{z\left(I_{p, \mu}^{a, c} f(z)\right)^{\prime}}{I_{p, \mu}^{a, c} f(z)}-p\right|-\operatorname{Re}\left(\frac{z\left(I_{p, \mu}^{a, c} f(z)\right)^{\prime}}{I_{p, \mu}^{a, c} f(z)}-p\right) \leq p-\alpha .
$$


R.M. El-Ashwah, A.Y. Lashin, A.E. El-Shirbiny - On Certain Subclass ...

We have

$$
\begin{aligned}
& \beta\left|\frac{z\left(I_{p, \mu}^{a, c} f(z)\right)^{\prime}}{I_{p, \mu}^{a, c} f(z)}-p\right|-\operatorname{Re}\left(\frac{z\left(I_{p, \mu}^{a, c} f(z)\right)^{\prime}}{I_{p, \mu}^{a, c} f(z)}-p\right) \\
\leq & (1+\beta)\left|\frac{z\left(I_{p, \mu}^{a, c} f(z)\right)^{\prime}}{I_{p, \mu}^{a, c} f(z)}-p\right| \\
\leq & (1+\beta)\left|\frac{p z^{p}+\sum_{k=n}^{\infty}(k+p) \Psi_{p, \mu}^{a, c}(k) a_{k+p} z^{k+p}}{z^{p}+\sum_{k=n}^{\infty} \Psi_{p, \mu}^{a, c}(k) a_{k+p} z^{k+p}}-p\right| \\
\leq & (1+\beta) \frac{\sum_{k=n}^{\infty} k \Psi_{p, \mu}^{a, c}(k) a_{k+p} z^{k}}{1-\sum_{k=n}^{\infty} \Psi_{p, \mu}^{a, c}(k) a_{k+p} z^{k}} .
\end{aligned}
$$

The last expression is bounded by $(p-\alpha)$, if

$$
\sum_{k=n}^{\infty}[k(1+\beta)+(p-\alpha)] \Psi_{p, \mu}^{a, c}(k)\left|a_{k+p}\right| \leq(p-\alpha),
$$

and hence the proof is completed.

Theorem 2. A necessary and sufficient condition for a function $f(z)$ of the form (4) to be in $U S_{p}^{*} T(n, a, c ; \mu, \alpha, \beta)$ is

$$
\sum_{k=n}^{\infty}[k(1+\beta)+(p-\alpha)] \Psi_{p, \mu}^{a, c}(k)\left|a_{k+p}\right| \leq(p-\alpha) .
$$

Proof. The sufficient condition follows from Theorem 1. To prove the necessity, let $f \in U S_{p}^{*} T(n, a, c ; \mu, \alpha, \beta)$ and $z$ is real, then

$$
\begin{aligned}
& \frac{p-\sum_{k=n}^{\infty}(k+p) \Psi_{p, \mu}^{a, c}(k) a_{k+p} z^{k}}{1-\sum_{k=n}^{\infty} \Psi_{p, \mu}^{a, c}(k) a_{k+p} z^{k}}-\alpha \\
\geq & \beta\left|\frac{p-\sum_{k=n}^{\infty}(k+p) \Psi_{p, \mu}^{a, c}(k) a_{k+p} z^{k}-p+\sum_{k=n}^{\infty} p \Psi_{p, \mu}^{a, c}(k) a_{k+p} z^{k}}{1-\sum_{k=n}^{\infty} \Psi_{p, \mu}^{a, c}(k) a_{k+p} z^{k}}\right| .
\end{aligned}
$$

Let $z \rightarrow 1^{-}$, we obtain

$$
\frac{p-\sum_{k=n}^{\infty}(k+p) \Psi_{p, \mu}^{a, c}(k) a_{k+p}}{1-\sum_{k=n}^{\infty} \Psi_{p, \mu}^{a, c}(k) a_{k+p}}-\alpha \geq \beta\left|\frac{-\sum_{k=n}^{\infty} k \Psi_{p, \mu}^{a, c}(k) a_{k+p}}{1-\sum_{k=n}^{\infty} \Psi_{p, \mu}^{a, c}(k) a_{k+p}}\right|
$$

or, equivalently

$$
p-\sum_{k=n}^{\infty}(k+p) \Psi_{p, \mu}^{a, c}(k)\left|a_{k+p}\right|-\alpha\left(1-\sum_{k=n}^{\infty} \Psi_{p, \mu}^{a, c}(k)\left|a_{k+p}\right|\right) \geq \beta \sum_{k=n}^{\infty} k \Psi_{p, \mu}^{a, c}(k)\left|a_{k+p}\right| .
$$


R.M. El-Ashwah, A.Y. Lashin, A.E. El-Shirbiny - On Certain Subclass ...

Thus, we have

$$
\sum_{k=n}^{\infty}[k(1+\beta)+(p-\alpha)] \Psi_{p, \mu}^{a, c}(k)\left|a_{k+p}\right| \leq(p-\alpha) .
$$

Then the proof is completed.

Corollary 3. If $f(z)$ of the form (4) is in $U S_{p}^{*} T(n, a, c ; \mu, \alpha, \beta)$, then

$$
a_{p+k} \leq \frac{(p-\alpha)}{[k(1+\beta)+(p-\alpha)] \Psi_{p, \mu}^{a, c}(k)}, \quad(k \geq n, n \in \mathbb{N}) .
$$

with equality only for the function

$$
f(z)=z^{p}-\frac{(p-\alpha)}{[k(1+\beta)+p-\alpha] \Psi_{p, \mu}^{a, c}(k)} z^{p+k}, \quad(k \geq n, n \in \mathbb{N}) .
$$

\section{HoldeR's inEQUALITY}

For function $f_{j}(z) \in T(p, n)$ are given by

$$
f_{j}(z)=z^{p}-\sum_{k=n}^{\infty} a_{k+p, j} z^{k+p} \quad\left(a_{k+p, j} \geq 0, j=1,2,3, \ldots, m\right) .
$$

Now, we define the modified Hadmard product of $f_{j}(z)$ and the generalization of the modified Hadmard product as follows

$$
G_{m}(z)=z^{p}-\sum_{k=n}^{\infty}\left(\prod_{j=1}^{m} a_{k+p, j}\right) z^{k+p}
$$

and

$$
H_{m}(z)=z^{p}-\sum_{k=n}^{\infty}\left(\prod_{j=1}^{m} a_{k+p, j}^{q_{j}}\right) z^{k+p}, \quad\left(q_{j}>0, j=1,2,3, \ldots, m\right) .
$$

(i) For $m=2$, then $G_{2}(z)=\left(f_{1} * f_{2}\right)(z)$.

(ii) For $q_{j}=1$, we have $G_{m}(z)=H_{m}(z)$. 
R.M. El-Ashwah, A.Y. Lashin, A.E. El-Shirbiny - On Certain Subclass ...

Further, for functions $f_{j}(z)(j=1,2, \ldots, m)$, the familiar Holder's inequality assumes the following form

$\sum_{k=n}^{\infty}\left(\prod_{j=1}^{m} a_{k+p, j}\right) \leq \prod_{j=1}^{m}\left(\sum_{k=n}^{\infty}\left(a_{k+p, j}\right)^{q_{j}}\right)^{\frac{1}{q_{j}}}, \quad\left(q_{j}>1, \sum_{j=1}^{m} \frac{1}{q_{j}} \geq 1, j=1,2,3, \ldots, m\right)$.

Recently, Nishiwaki and Owa [15] have studied some results of Holder's inequalities for a subclass of $p$-valent starlike and convex function.

Theorem 4. Let $f_{j}(z) \in U S_{p}^{*} T\left(n, a, c ; \mu, \alpha_{j}, \beta\right)(j=1,2,3, \ldots, m)$, then $H_{m}(z) \in$ $U S_{p}^{*} T(n, a, c ; \mu, \eta, \beta)$,

$$
\eta \leq p-\frac{[k(1+\beta)] \prod_{j=1}^{m}\left(p-\alpha_{j}\right)^{s_{j}}}{\prod_{j=1}^{m}\left[k(1+\beta)+\left(p-\alpha_{j}\right)\right]^{s_{j}}\left[\Psi_{p, \mu}^{a, c}(k)\right]^{s_{j-1}}-\prod_{j=1}^{m}\left(p-\alpha_{j}\right)^{s_{j}}},
$$

where $k \geq n, s_{j} \geq \frac{1}{q_{j}}, q_{j}>1, \sum_{j=1}^{m} \frac{1}{q_{j}} \geq 1 ; j=1,2,3, \ldots, m$.

Proof. Let $f_{j}(z) \in U S_{p}^{*} T\left(n, a, c ; \mu, \alpha_{j}, \beta\right)$, then

$$
\sum_{k=n}^{\infty} \frac{\left[k(1+\beta)+\left(p-\alpha_{j}\right)\right] \Psi_{p, \mu}^{a, c}(k)}{\left(p-\alpha_{j}\right)} a_{k+p, j} \leq 1
$$

which implies

$$
\left(\sum_{k=n}^{\infty} \frac{\left[k(1+\beta)+\left(p-\alpha_{j}\right)\right] \Psi_{p, \mu}^{a, c}(k)}{\left(p-\alpha_{j}\right)} a_{k+p, j}\right)^{\frac{1}{q_{j}}} \leq 1, \quad\left(q_{j}>1, \quad \sum_{j=1}^{m} \frac{1}{q_{j}} \geq 1\right) .
$$

From (13), we have

$$
\prod_{j=1}^{m}\left(\sum_{k=n}^{\infty} \frac{\left[k(1+\beta)+\left(p-\alpha_{j}\right)\right] \Psi_{p, \mu}^{a, c}(k)}{\left(p-\alpha_{j}\right)} a_{k+p, j}\right)^{\frac{1}{q_{j}}} \leq 1 .
$$

Applying Holder's inequality, we find that

$$
\sum_{k=n}^{\infty}\left[\prod_{j=1}^{m}\left(\frac{\left[k(1+\beta)+\left(p-\alpha_{j}\right)\right] \Psi_{p, \mu}^{a, c}(k)}{\left(p-\alpha_{j}\right)}\right)^{\frac{1}{q_{j}}}\left(a_{k+p, j}\right)^{\frac{1}{q_{j}}}\right] \leq 1 .
$$


R.M. El-Ashwah, A.Y. Lashin, A.E. El-Shirbiny - On Certain Subclass ...

Thus, we have to determine the largest $\eta$ such that

$$
\sum_{k=n}^{\infty} \frac{[k(1+\beta)+(p-\eta)] \Psi_{p, \mu}^{a, c}(k)}{(p-\eta)}\left(\prod_{j=1}^{m} a_{k+p, j}^{s_{j}}\right) \leq 1 .
$$

That is

$$
\sum_{k=n}^{\infty} \frac{[k(1+\beta)+(p-\eta)] \Psi_{p, \mu}^{a, c}(k)}{(p-\eta)}\left(\prod_{j=1}^{m} a_{k+p, j}^{s_{j}}\right) \leq \sum_{k=n}^{\infty}\left[\prod_{j=1}^{m}\left(\frac{\left[k(1+\beta)+\left(p-\alpha_{j}\right)\right] \Psi_{p, \mu}^{a, c}(k)}{\left(p-\alpha_{j}\right)}\right)^{\frac{1}{q_{j}}}\left(a_{k+p, j}\right)^{\frac{1}{q_{j}}}\right] .
$$

Therefore, we need to find the largest $\eta$ such that

$\frac{[k(1+\beta)+(p-\eta)] \Psi_{p, \mu}^{a, c}(k)}{(p-\eta)}\left(\prod_{j=1}^{m}\left(a_{k+p, j}\right)^{s_{j}-\frac{1}{q_{j}}}\right) \leq \prod_{j=1}^{m}\left(\frac{\left[k(1+\beta)+\left(p-\alpha_{j}\right)\right] \Psi_{p, \mu}^{a, c}(k)}{\left(p-\alpha_{j}\right)}\right)^{\frac{1}{q_{j}}}, \quad(k \geq n)$.

Since

$$
\prod_{j=1}^{m}\left(\frac{\left[k(1+\beta)+\left(p-\alpha_{j}\right)\right] \Psi_{p, \mu}^{a, c}(k)}{\left(p-\alpha_{j}\right)}\right)^{s_{j}-\frac{1}{q_{j}}}\left(a_{k+p, j}\right)^{s_{j}-\frac{1}{q_{j}}} \leq 1, \quad\left(s_{j}-\frac{1}{q_{j}} \geq 0\right) .
$$

We see that,

$$
\prod_{j=1}^{m}\left(a_{k+p, j}\right)^{s_{j}-\frac{1}{q_{j}}} \leq \frac{1}{\prod_{j=1}^{m}\left(\frac{\left[k(1+\beta)+\left(p-\alpha_{j}\right)\right] \Psi_{p, \mu}^{a, c}(k)}{\left(p-\alpha_{j}\right)}\right)^{s_{j}-\frac{1}{q_{j}}}} .
$$

This implies that

$$
\frac{[k(1+\beta)+(p-\eta)] \Psi_{p, \mu}^{a, c}(k)}{(p-\eta)} \leq \frac{\prod_{j=1}^{m}\left[\left[k(1+\beta)+\left(p-\alpha_{j}\right)\right] \Psi_{p, \mu}^{a, c}(k)\right]^{s_{j}}}{\prod_{j=1}^{m}\left(p-\alpha_{j}\right)^{s_{j}}} .
$$

Then

$$
\eta \leq p-\frac{k(1+\beta) \prod_{j=1}^{m}\left(p-\alpha_{j}\right)^{s_{j}}}{\prod_{j=1}^{m}\left[k(1+\beta)+\left(p-\alpha_{j}\right)\right]^{s_{j}}\left[\Psi_{p, \mu}^{a, c}(k)\right]^{s_{j-1}}-\prod_{j=1}^{m}\left(p-\alpha_{j}\right)^{s_{j}}}
$$

This completes the proof of the theorem.

Remark 1. Putting $a=c, \mu=1, \beta=0$ in Theorem 4, we obtain the corresponding result obtained by Nishiwaki and Owa [15];

Corollary 5. Let $f_{j}(z) \in U S_{p}^{*} T\left(n, a, c ; \mu, \alpha_{j}, \beta\right)(j=1,2,3, \ldots, m)$, then $H_{m}(z) \in$ $U S_{p}^{*} T(n, a, c ; \mu, \eta, \beta)$ with

$$
\eta \leq p-\frac{n(1+\beta) \prod_{j=1}^{m}\left(p-\alpha_{j}\right)^{s_{j}}}{\left[\Psi_{p, \mu}^{a, c}(n)\right]^{r-1} \prod_{j=1}^{m}\left[n(1+\beta)+\left(p-\alpha_{j}\right)\right]^{s_{j}}-\prod_{j=1}^{m}\left(p-\alpha_{j}\right)^{s_{j}}}
$$

where $r=\sum_{j=1}^{m} s_{j}>1+\frac{p-\alpha}{n(1+\beta)}, s_{j} \geq \frac{1}{q_{j}}, q_{j}>1, \sum_{j=1}^{m} \frac{1}{q_{j}} \geq 1 ; j=1,2,3, \ldots, m$. 
R.M. El-Ashwah, A.Y. Lashin, A.E. El-Shirbiny - On Certain Subclass ...

Putting $\alpha_{j}=\alpha$ in Corollary 5 we obtain the following corollary.

Corollary 6. Let $f_{j}(z) \in U S_{p}^{*} T\left(n, a, c ; \mu, \alpha_{j}, \beta\right)(j=1,2,3, \ldots, m)$, then $H_{m}(z) \in$ $U S_{p}^{*} T(n, a, c ; \mu, \eta, \beta)$ with

$$
\eta \leq p-\frac{[n(1+\beta)](p-\alpha)^{r}}{[n(1+\beta)+(p-\alpha)]^{r}\left[\Psi_{p, \mu}^{a, c}(n)\right]^{r-1}-(p-\alpha)^{r}}
$$

where $r=\sum_{j=1}^{m} s_{j}>1+\frac{p-\alpha}{n(1+\beta)}, s_{j} \geq \frac{1}{q_{j}}, q_{j}>1, \sum_{j=1}^{m} \frac{1}{q_{j}} \geq 1 ; j=1,2,3, \ldots, m$.

Example 1. Let $f_{j}(z)(j=1,2,3, \ldots, m)$ define as follows

$f_{j}(z)=z^{p}-\frac{(p-\alpha)}{[n(1+\beta)+(p-\alpha)] \Psi_{p, \mu}^{a, c}(n)} \epsilon z^{n+p}-\frac{(p-\alpha)}{[(n+j)(1+\beta)+(p-\alpha)] \Psi_{p, \mu}^{a, c}(n+j)} \epsilon_{j} z^{n+p+j}$,
$\left(\epsilon+\epsilon_{j} \leq 1\right)$,

then $H_{m}(z) \in U S_{p}^{*} T(n, a, c ; \mu, \eta, \beta)$ with

$$
\eta \leq p-\frac{[n(1+\beta)](p-\alpha)^{r}}{[n(1+\beta)+(p-\alpha)]^{r}\left[\Psi_{p, \mu}^{a, c}(n)\right]^{r-1}-(p-\alpha)^{r}} .
$$

Since

$$
\begin{aligned}
f_{j}(z)= & z^{p}-\frac{(p-\alpha)}{[n(1+\beta)+(p-\alpha)] \Psi_{p, \mu}^{a, c}(n)} \epsilon z^{n+p}-\frac{(p-\alpha)}{[(n+j)(1+\beta)+(p-\alpha)] \Psi_{p, \mu}^{a, c}(n+j)} \epsilon_{j} z^{n+p+j}, \\
& \left(\epsilon+\epsilon_{j} \leq 1, j=1,2,3, \ldots, m\right),
\end{aligned}
$$

we have

$$
\begin{aligned}
\sum_{k=n}^{\infty} \frac{[k(1+\beta)+(p-\alpha)] \Psi_{p, \mu}^{a, c}(k)}{(p-\alpha)} a_{k+p}= & \frac{[n(1+\beta)+(p-\alpha)] \Psi_{p, \mu}^{a, c}(n)}{(p-\alpha)} \epsilon a_{n+p} \\
& +\frac{[(n+j)(1+\beta)+(p-\alpha)] \Psi_{p, \mu}^{a, c}(n+j)}{(p-\alpha)} \epsilon_{j} a_{n+p+j} \\
= & \epsilon+\epsilon_{j} \leq 1 .
\end{aligned}
$$

Then $f_{j}(z) \in U S_{p}^{*} T(n, a, c ; \mu, \alpha, \beta)$ and we have

$$
H_{m}(z)=z^{p}-\left(\frac{(p-\alpha)}{[n(1+\beta)+(p-\alpha)] \Psi_{p, \mu}^{a, c}(n)} \epsilon\right)^{r} z^{n+p}
$$

and $H_{m}(z) \in U S_{p}^{*} T(n, a, c ; \mu, \eta, \beta)$. 
R.M. El-Ashwah, A.Y. Lashin, A.E. El-Shirbiny - On Certain Subclass ...

\section{Modified HadAmard PROduCtS}

Let the functions $f_{i}(z)(i=1,2)$ be defined by

$$
f_{i}(z)=z^{p}-\sum_{k=n}^{\infty} a_{k+p, i} z^{k+p}
$$

The modified Hadamard product of $f_{1}(z)$ and $f_{2}(z)$ is defined by

$$
\left(f_{1} * f_{2}\right)(z)=z^{p}-\sum_{k=n}^{\infty} a_{k+p, 1} a_{k+p, 2} z^{k+p} .
$$

Corollary 7. Let the functions $f_{i}(z)(i=1,2)$ defined by $(14)$ be in the class $U S_{p}^{*} T\left(n, a, c ; \mu, \alpha_{1}, \beta\right)$ and $U S_{p}^{*} T\left(n, a, c ; \mu, \alpha_{2}, \beta\right)$, then

$\left(f_{1} * f_{2}\right)(z) \in U S_{p}^{*} T(n ; a, c ; \mu, \delta, \beta)$ where

$\delta \leq p-\frac{n(1+\beta)\left(p-\alpha_{1}\right)\left(p-\alpha_{2}\right)}{\left[n(1+\beta)+\left(p-\alpha_{1}\right)\right]\left[n(1+\beta)+\left(p-\alpha_{2}\right)\right] \Psi_{p, \mu}^{a, c}(n)-\left(p-\alpha_{1}\right)\left(p-\alpha_{2}\right)} \quad(z \in U ; n \in \mathbb{N})$.

Corollary 8. Let the functions $f_{i}(z)(i=1,2)$ defined by (14) be in the class $U S_{p}^{*} T(n, a, c ; \mu, \alpha, \beta)$ then $\left(f_{1} * f_{2}\right)(z) \in U S_{p}^{*} T(n, a, c ; \mu, \delta, \beta)$ where

$$
\delta \leq p-\frac{n(1+\beta)(p-\alpha)^{2}}{\Psi_{p, \mu}^{a, c}(n)[n(1+\beta)+(p-\alpha)]^{2}-(p-\alpha)^{2}} \quad(z \in U ; n \in \mathbb{N}) .
$$

Theorem 9. Let the functions $f_{i}(z)(i=1,2)$ defined by (14) be in the class $U S_{p}^{*} T(n, a, c ; \mu, \alpha, \beta)$, then $h(z)=z^{p}-\sum_{k=p+1}^{\infty}\left(a_{k, 1}^{2}+a_{k, 2}^{2}\right) z^{p}$ belongs to the class $U S_{p}^{*} T(n, a, c ; \mu, \delta, \beta)$ where

$$
\delta=\Omega(n) \leq p-\frac{2 n(1+\beta)(p-\alpha)^{2}}{[n(1+\beta)+(p-\alpha)]^{2} \Psi_{p, \mu}^{a, c}(n)-2(p-\alpha)^{2}} \quad(z \in U, n \in \mathbb{N}) .
$$

Proof. To prove the theorem, we need to find the largest $\delta$ such that

$$
\sum_{k=p+1}^{\infty} \frac{[k(1+\beta)+(p-\delta)] \Psi_{p, \mu}^{a, c}(k)}{(p-\delta)}\left(a_{k, 1}^{2}+a_{k, 2}^{2}\right) \leq 1
$$

Hence

$\sum_{k=n}^{\infty}\left\{\frac{[k(1+\beta)+(p-\alpha)] \Psi_{p, \mu}^{a, c}(k)}{(p-\alpha)}\right\}^{2} a_{k, i}^{2} \leq\left\{\sum_{k=n}^{\infty} \frac{[k(1+\beta)+(p-\alpha)] \Psi_{p, \mu}^{a, c}(k)}{(p-\alpha)} a_{k, i}\right\}^{2} \leq 1, \quad(i=1,2)$. 
R.M. El-Ashwah, A.Y. Lashin, A.E. El-Shirbiny - On Certain Subclass ...

Then

$$
\sum_{k=n}^{\infty} \frac{1}{2}\left[\frac{[k(1+\beta)+(p-\alpha)]}{(p-\alpha)} \Psi_{p, \mu}^{a, c}(k)\right]^{2}\left(a_{k, 1}^{2}+a_{k, 2}^{2}\right) \leq 1,
$$

and (15) is true if

$$
\sum_{k=n}^{\infty} \frac{[k(1+\beta)+(p-\delta)] \Psi_{p, \mu}^{a, c}(k)}{(p-\delta)}\left(a_{k, 1}^{2}+a_{k, 2}^{2}\right) \leq \sum_{k=n}^{\infty} \frac{1}{2}\left[\frac{[k(1+\beta)+(p-\alpha)] \Psi_{p, \mu}^{a, c}(k)}{(p-\alpha)}\right]^{2}\left(a_{k, 1}^{2}+a_{k, 2}^{2}\right) .
$$

If

$$
\frac{[k(1+\beta)+(p-\delta)]}{(p-\delta)} \leq \frac{[k(1+\beta)+(p-\alpha)]^{2}}{2(p-\alpha)^{2}} \Psi_{p, \mu}^{a, c}(k),
$$

then

$$
\delta \leq \Omega(k)=p-\frac{2 k(1+\beta)(p-\alpha)^{2}}{[k(1+\beta)+(p-\alpha)]^{2} \Psi_{p, \mu}^{a, c}(k)-2(p-\alpha)^{2}}, \quad(k \geq n, n \in \mathbb{N})
$$

which is an increasing function of $k \geq n, 0 \leq \alpha<p, p \in \mathbb{N}, 0<\beta \leq 1$.

Then

$$
\delta=\Omega(n) \leq p-\frac{2 n(1+\beta)(p-\alpha)^{2}}{[n(1+\beta)+(p-\alpha)]^{2} \Psi_{p, \mu}^{a, c}(n)-2(p-\alpha)^{2}} .
$$

The proof is completed.

\section{Closure Properties under Integral Operators}

In this section, we discuss some preserving integral operators. We recall here the generalized Komatu integral operator (see [13]) define by

$$
\begin{aligned}
K(z) & =\frac{(\gamma+p)^{d}}{\Gamma(d) z^{\gamma}} \int_{0}^{z} t^{\gamma-1}\left(\log \frac{z}{t}\right)^{d-1} f(t) d t \quad(f(z) \in T(n, p)) \\
& =z^{p}-\sum_{k=n}^{\infty}\left(\frac{\gamma+p}{\gamma+k+p}\right)^{d} a_{k+p} z^{k+p} \quad(d \geq 0 ; \gamma>-p ; z \in U)
\end{aligned}
$$

Also the generalized Jung-Kim-Srivastava operator (see[11]) define by

$$
\begin{aligned}
I(z) & =Q_{\gamma, p}^{d} f(z)=\left(\begin{array}{c}
p+d+\gamma-1 \\
p+\gamma-1
\end{array}\right) \frac{d}{z^{\gamma}} \int_{0}^{z} t^{\gamma-1}\left(1-\frac{t}{z}\right)^{d-1} f(t) d t \quad(f(z) \in T(n, p)) \\
& =z^{p}-\sum_{k=n}^{\infty} \frac{\Gamma(p+k+\gamma) \Gamma(p+\gamma+d)}{\Gamma(p+k+\gamma+d) \Gamma(p+\gamma)} a_{k+p} z^{k+p} \quad(d \geq 0 ; \gamma>-p ; z \in U) .
\end{aligned}
$$


R.M. El-Ashwah, A.Y. Lashin, A.E. El-Shirbiny - On Certain Subclass ...

Theorem 10. Let $d>0, \gamma>-p$ and $f(z) \in U S_{p}^{*} T(n, a, c ; \mu, \alpha, \beta)$, then $K(z)$ defined by (17) belongs to $U S_{p}^{*} T(n, a, c ; \mu, \alpha, \beta)$.

Proof. Let $f(z) \in U S_{p}^{*} T(n, a, c ; \mu, \alpha, \beta)$ defined by (4), and $K(z)$ defined by (17) Then $K(z) \in U S_{p}^{*} T(n, a, c ; \mu, \alpha, \beta)$ if

$$
\sum_{k=n}^{\infty} \frac{[k(1+\beta)+(p-\alpha)] \Psi_{p, \mu}^{a, c}(k)}{(p-\alpha)}\left(\frac{\gamma+p}{\gamma+k+p}\right)^{d} a_{k+p} \leq 1 .
$$

Now, from Theorem $2, f(z) \in U S_{p}^{*} T(n, a, c ; \mu, \alpha, \beta)$ if and only if

$$
\sum_{k=n}^{\infty} \frac{[k(1+\beta)+(p-\alpha)] \Psi_{p, \mu}^{a, c}(k)}{(p-\alpha)} a_{k+p} \leq 1 .
$$

Since $\frac{\gamma+p}{\gamma+k+p} \leq 1$, for $k \geq n$, then (19) holds true. Therefore $K(z) \in U S_{p}^{*} T(n, a, c ; \mu, \alpha, \beta)$.

Theorem 11. Let $d>0, \gamma>-p$ and $f(z) \in U S_{p}^{*} T(n, a, c ; \mu, \alpha, \beta)$, then $K(z)$ defined by (17) is $p$-valent in the disk $|z|<R_{1}$, where

$$
R_{1}=\inf _{k}\left\{\frac{p[k(1+\beta)+(p-\alpha)](\gamma+k+p)^{d}}{(k+p)(p-\alpha)(\gamma+p)^{d}} \Psi_{p, \mu}^{a, c}(k)\right\}^{\frac{1}{k}}
$$

Proof. In order to prove the assertion, it is enough to show that

$$
\left|\frac{K^{\prime}(z)}{z^{p-1}}-p\right| \leq p
$$

Now, in view of (21), we get

$$
\begin{aligned}
\left|\frac{K^{\prime}(z)}{z^{p-1}}-p\right| & =\left|-\sum_{k=n}^{\infty}(k+p)\left(\frac{\gamma+p}{\gamma+k+p}\right)^{d} a_{k+p} z^{k+p}\right| \\
& \leq \sum_{k=n}^{\infty}(k+p)\left(\frac{\gamma+p}{\gamma+k+p}\right)^{d} a_{k+p}\left|z^{k}\right| .
\end{aligned}
$$

This expression is bounded by $p$ if

$$
\sum_{k=n}^{\infty} \frac{(k+p)}{p}\left(\frac{\gamma+p}{\gamma+k+p}\right)^{d} a_{k+p}\left|z^{k}\right| \leq 1
$$


R.M. El-Ashwah, A.Y. Lashin, A.E. El-Shirbiny - On Certain Subclass ...

Since $f(z) \in U S_{p}^{*} T(n, a, c ; \mu, \alpha, \beta)$, and from Theorem $2(22)$ holds if

$$
\frac{(k+p)}{p}\left(\frac{\gamma+p}{\gamma+k+p}\right)^{d} a_{k+p}\left|z^{k}\right| \leq \frac{[k(1+\beta)+(p-\alpha)] \Psi_{p, \mu}^{a, c}(k)}{(p-\alpha)} a_{n+p}, \quad(k \in \mathbb{N}) .
$$

That is

$$
|z| \leq\left\{\frac{p[k(1+\beta)+(p-\alpha)](\gamma+k+p)^{d}}{(k+p)(p-\alpha)(\gamma+p)^{d}} \Psi_{p, \mu}^{a, c}(k)\right\}^{\frac{1}{k}} .
$$

The result follows by setting $|z|=R_{1}$.

Following similar steps as in the proofs of Theorem 10 and Theorem 11, we have the following results for $I(z)$.

Theorem 12. Let $d>0, \gamma>-p$ and $f(z) \in U S_{p}^{*} T(n, a, c ; \mu, \alpha, \beta)$, then $I(z)$ defined by (18) belongs to $U S_{p}^{*} T(n, a, c ; \mu, \alpha, \beta)$.

Theorem 13. Let $d>0, c>-p$ and $f(z) \in U S_{p}^{*} T(n ; a, c ; \mu, \alpha, \beta)$. Then $I(z)$ defined by (18) is $p$-valent in the disk $|z|<R_{2}$, where

$$
R_{2}=\inf _{k}\left\{\frac{p[k(1+\beta)+(p-\alpha)] \Gamma(p+k+\gamma+d) \Gamma(p+\gamma)}{(k+p)(p-\alpha) \Gamma(p+k+\gamma) \Gamma(p+\gamma+d)} \Psi_{p, \mu}^{a, c}(k)\right\}^{\frac{1}{k}}
$$

\section{REFERENCES}

[1] M. K. Aouf, R. M. El-Ashwah and A. M. Abd-Eltawab, Some inclusion relationships of certain subclasses of p-valent functions associated with a family of integral operators, ISRN Math. Anal., Art., ID 384170, (2013), 1-8.

[2] R. M. EL-Ashwah and M. E. Drbuk, Subordination Properties of p-valent functions defined by linear operators, British J. Math. and comput. Sci., 4 (21), 2016, 3000-3013.

[3] S. D. Bernardi, Convex and starlike univalent functions, Trans. Amer. Math. Soc., 135 (1969), 429-446.

[4] R. Bharati, R. Paravatham and A. Swaminathan, On subclasses of uniformly convex functions and corresponding class of starlike functions, Tamkang J. Math., 28 (1997), no. 1, 17-32.

[5] B. C. Carlson and D. B. Shafer, Starlike and prestarlike Hypergeometric functions, J. Math. Anal., 15 (1984), no. 4, 737-745.

[6] J. H. Choi, M. Saigo and H. M. Srivastava, Some inclusion properties of a certain family of integral operators, J. Math. Anal. Appl., 276 (2002), 432-445. 
R.M. El-Ashwah, A.Y. Lashin, A.E. El-Shirbiny - On Certain Subclass ...

[7] A. W. Goodman, On uniformly convex functions, Ann. Polon. Math., 56 (1991), no. $1,87-92$.

[8] I. B. Jung, Y. C. Kim and H. M. Srivastava, The hardy space of analytic functions associated with certain parameter families of integral operators, J. Math. Anal. Appl., 176 (1993), 138-147.

[9] S. Kanas and A. Wisniowska, Conic regions and k-uniform convexity, J. Comput. Appl. Math., 105 (1999), no. 1-2, 327-336.

[10] S. M. Khairnar and M. More, On a subclass of multivalent - uniformly starlike and convex functions defined by a linear operator, IAENG Int. J. Appl. Math., Doi: 39:3, IJAM3-39-06-2009.

[11] V. Kiryakova, Generalized fractional calculus and applications, Pitman Research Notes in Mathematics Series, 301, John Willey and Sons, Inc. New York , 1994.

[12] R. J. Libera, Some classes of regular univalent functions, Proc. Amer. Math. Soc., 16 (1965), 755-758.

[13] J. L. Liu and S. Owa, Properties of certain integral operator, Int. J. Math. Sci., 3 (2004), no.1, 45-51.

[14] A. E. Livingston, On the radius of univalence of certain analytic functions, Proc. Amer. Math. Soc., 17 (1966), 352-357.

[15] J. Nishiwaki and S. Owa, An application of Holder's inequality for convolutions, J. Inequal. Pure Appl. Math., 10 (2009), no. 4, Art. 98, 1-7.

[16] K. I. Noor, On new classes of integral operators, J. Natural Geometry, 16 (1999), 71-80.

[17] K. I. Noor and M. A. Noor, On integral operators, J. Math. Anal. Appl., 238 (1999), 341-352.

[18] S. Owa, Some properties of certain multivalent functions, Appl. Math. Letters, 4(1991), no. 5, 79-83.

[19] D. A. Patil, N. K. Thakare, On convex hulls and extreme points of p-valent starlike and convex classes with applications, Bull. Math. Soc. Sci. Math. R. S. Romania(N. S.), 27 (1983), no. 2, 145-160.

[20] R. K. Raina and P. Sharma, Subordination properties of univalent functions involving a new class of operators, Electronic J. Math. Anal. Appl., 2 (2014), no. 1, $37-52$.

[21] R. K. Raina and P. Sharma, Subordination preserving properties associated with a class of operators, Le Matematiche, 68 (2013), 217-228.

[22] S. Ruscheweyh, New criteria for univalent functions, Proc. Amer. Math. Soc., 49 (1975), 109-115. 
R.M. El-Ashwah, A.Y. Lashin, A.E. El-Shirbiny - On Certain Subclass ...

[23] H. Saitoh, S. Owa, T. Sekine, M. Nunokawa and R. Yamakawa, An application of certain integral operator, Appl. Math. Letters, 5 (1992), 21-24.

R.M. El-Ashwah

Department of Mathematics, Faculty of science, Damietta University,

Damietta, Egypt.

email: r_elashwah@yahoo.com

A.Y. Lashin

Department of Mathematics, Faculty of Science, Mansoura University, Mansoura 35516, Egypt.

email: aylashin@mans.edu.eg

A.E. El-Shirbiny

Department of Mathematics, Faculty of science,

Damietta University,

Damietta, Egypt.

email:amina.ali66@yahoo.com 